\title{
ADOLESCENTES EN LAS AULAS: LA IRRUPCIÓN DE LA DIFERENCIA Y EL FIN DE LA EXPANSIÓN EDUCATIVA
}

\author{
NÉSTOR LÓPEZ
}

\begin{abstract}
RESUMEN: El artículo se inicia con una revisión de las tendencias recientes en la escolarización de los adolescentes en América Latina, poniendo énfasis en analizar en qué medida la expansión de la escolarización desde inicios de los 90 se tradujo en una reducción importante de las desigualdades en el acceso a la educación, y al mismo tiempo cómo la desaceleración del proceso de escolarización que se vive en este último lustro reinstala el desafío de las desigualdades como elemento central en la agenda educativa. La segunda parte del artículo plantea algunas hipótesis en torno a las causas de esa desaceleración visible en los procesos de universalización del acceso a la escuela, enfatizando en aquellas que centran la atención en las desigualdades que resultan de la imposibilidad de los sistemas educativos de operar en contextos de creciente diversidad cultural e identitaria, evidenciando la persistencia de múltiples mecanismos cotidianos y naturalizados de discriminación en las prácticas de las instituciones escolares
\end{abstract}

Palabras clave: Educación. Desigualdad. Diversidad. Adolescentes.

\section{AdolescentS IN THE CLASSROOM: THE EMERGENCE OF DIVERSITY AND THE END OF THE EXPANSION OF EDUCATION}

ABSTRACT: The article begins by reviewing recent trends in the education of adolescents in Latin America. Specifically, the analysis is focused on to which extent the expansion of education since the early 1990's resulted in an important reduction of inequalities in the access to education and at the same time how the deceleration of the education process, that has been going on over the last five years, reinstalls the challenge of inequalities in the core of the educational agenda. The second part of the article suggests some hypothesis regarding the causes for this deceleration that may be seen in the universalization process of access to education. A special attention is paid to those hypothesis focusing on inequalities that result from the educational system's difficulties in operating in contexts of increasing cultural and identity diversity that clearly shows the persistence of multiple daily and common discriminatory practices at schools.

Key words: Education. Inequality. Diversity. Adolescents.

Sociólogo e investigador del Instituto Internacional de Planeamiento de la Educación (IIPE) da Unesco, Buenos Aires. E-mail: n.lopez@iipe-buenosaires.org.ar 


\title{
Adolescents EN COURS: L'IRRUPTION DE LA DIFFERENCE ET LA FIN DE L'EXPANSION ÉDUCATIVE
}

\begin{abstract}
RÉSUMÉ: L'article commence avec une révision des tendances récentes dans la scolarisation des adolescents en Amérique Latine, en mettant l'emphase sur l'analyse de la mesure dans laquelle l'expansion de la scolarisation depuis le début des années 1990 s'est traduite en une réduction importante des inégalités pour l'accès à l'éducation, et en même temps comment la désaccélération du processus de scolarisation qui a lieu à ce niveau réinstalle le défi des inégalités comme élément central dans l'agenda éducatif. La seconde partie de l'article présente quelques hypothèses autour des causes de cette désacélération visible dans les processus d'universalisation de l'accès à l'école, emphatisant sur celles qui centrent leur attention sur les inégalités qui résultent de l'impossibilité des systèmes éducatifs d'opérer en des contextes de croissante diversité culturelle et identitaire, mettant aussi en évidence la persistence de multiples mécanismes quotidiens et naturalisés de discrimination dans les pratiques des institutions scolaires.
\end{abstract}

Mots-clés: Éducation. Inégalité. Diversité. Adolescents.

\section{Introducción}

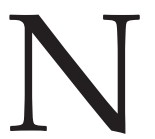

uestras sociedades son cada vez más difíciles de habitar. Las últimas décadas representaron una sucesión de profundos cambios políticos, económicos, sociales y culturales que fueron reconfigurando el mundo en que vivimos, y que lejos de tender hacia un nuevo punto de equilibrio, van desencadenando nuevos procesos de renovación permanente, los cuales adquieren cada vez mayor velocidad. Sin la pretensión de desarrollar estos temas, quisiera que en el momento de avanzar en la lectura de este texto se tenga en mente un breve listado de fenómenos ya largamente estudiados por muchos analistas, y que en su articulación configuran un nuevo mundo. Me refiero a los procesos de globalización económica y cultural, a la irrupción de internet como un elemento que atraviesa radicalmente la vida cotidiana, el carácter difuso que adquiere el límite entre lo material y lo digital, entre el espacio geográfico y el mundo virtual, el divorcio entre contenido y soporte - que pone en crisis, entre otras cosas, a las grandes industrias del mundo de la cultura -, la ampliación del espectro de opciones en la construcción de la identidad, la crisis de las representaciones en los modelos clásicos de funcionamiento de las democracias, la irrupción de los jóvenes como nuevos actores políticos y sociales tanto en los países de América Latina como en Europa o África del Norte -, entre otros. Listado arbitrario que podría ampliarse con elementos que refieren a aspectos muy diversos de la vida social, pero que alcanza para recordarnos que el mundo hoy ya no es lo que fue hace dos o tres décadas atrás.

Para poder vivir en este nuevo mundo se necesitan muchos recursos. El ejercicio de una ciudadanía plena, el desarrollo de cada uno de nosotros como sujetos 
íntegros y libres o la construcción de una identidad disfrutable se ven entorpecidos si no se cuenta con la capacidad de comprender y de discernir sobre lo que ocurre en nuestro entorno, y la posibilidad de constituirse en un ser productivo se ve limitada sin la adquisición de un conjunto cada vez mayor de saberes y destrezas. Los sistemas educativos se ven fuertemente interpelados por la demanda social de estos nuevos recursos, y paulatinamente han ido incorporando cambios orientados a dar respuesta a ella. Uno de los cambios más significativos que se dieron a lo largo de toda la geografía de la región es la redefinición de lo que es hoy la educación básica. Si en algún momento la enseñanza primaria fue concebida como la educación básica necesaria, hoy ya se asume que seis o siete años de escolaridad no son suficientes para poder garantizar a un sujeto este complejo conjunto de recursos. Así, la educación media pasó a formar parte de esta educación fundamental, y como consecuencia de ello la gran mayoría de los países han ampliado el ciclo de escolarización obligatoria, llegando en varios casos a 12 o 13 años.

Y como resultado de este cambio irrumpe un nuevo actor en la dinámica de los sistemas educativos: los adolescentes. Históricamente, para ellos la carrera educativa era una opción; finalizado el nivel primario podían avanzar en las diferentes modalidades del nivel medio, o retirarse al mundo del trabajo. Hoy, en cambio, esa educación media es un derecho irrenunciable, y una obligación para la sociedad y para los Estados. Aquel joven que podía ser expulsado hoy se constituye en un sujeto de derecho, y el Estado asume el compromiso de garantizarle ese derecho. Visto desde la perspectiva de la escuela, la inclusión del nivel medio como parte de la educación básica obligatoria representa un desafío extremadamente complejo: una institución que fue concebida como un instrumento de selección y clasificación, hoy debe transformarse en un espacio de inclusión social y formación de ciudadanos.

En este texto me propongo hacer una revisión de las tendencias recientes en la escolarización de los adolescentes en la región, poniendo énfasis en analizar en qué medida la expansión de la escolarización desde inicios de los 90 se tradujo en una reducción importante de las desigualdades en el acceso a la educación, y al mismo tiempo cómo la desaceleración - ¿o el fin de la expansión? - que se vive en este último lustro reinstala el desafío de las desigualdades como elemento central en la agenda educativa. En la segunda parte retomo algunas notas de estudios que estamos realizando en estos momentos en el IIPE-Unesco, en Buenos Aires, para plantear algunas hipótesis en torno a las causas de esa desaceleración visible en los procesos de universalización del acceso a la escuela, enfatizando en aquellas que centran la atención en las desigualdades que resultan de la imposibilidad de los sistemas educativos de operar en contextos de creciente diversidad cultural e identitaria, evidenciando la persistencia de múltiples mecanismos cotidianos y naturalizados de discriminación en las prácticas de las instituciones escolares. 


\section{¿El fin de la expansión educativa?}

Comienzo haciendo una revisión básica de algunos datos sobre la situación educativa de adolescentes de entre 15 y 17 años de edad en 18 países de América Latina. Un primer elemento a destacar es un aumento de su escolarización a lo largo de la última década. Tal como puede verse en la tabla 1 - en el anexo 1 -, los valores que tomaban las tasas de escolarización de estos adolescentes en el año 2000 promediaban en un $68 \%$, en tanto que hacia el año 2010 ese promedio ascendía al $75.8 \%$. Salvo en Nicaragua (donde se verifica una leve caída de la escolarización) y en Uruguay (donde el crecimiento es nulo), casi la totalidad de los países muestran un incremento en sus niveles de escolarización de los adolescentes. En algunos casos, como Guatemala o Costa Rica, ese crecimiento fue cercano al $40 \%$.

Este proceso de expansión del acceso a la escuela de los jóvenes fue más intenso en los países que a inicios de la década tenían las menores tasas de escolarización y entre los adolescentes de los estratos sociales más bajos, dando como resultado un doble efecto. Por un lado, una reducción de las disparidades entre países, achicando la brecha entre el valor más alto (Chile) y el más bajo (Guatemala en el año 2000 y Honduras en el 2010) de 2.15 a 1.7. Por el otro, una reducción de las desigualdades en el acceso dentro de cada uno de los países. Salvo Perú, todos los países de la región experimentan una fuerte reducción de la distancia entre las tasas de escolarización de los adolescentes de los estratos sociales más altos y las de los más bajos. También está documentada en diversas publicaciones del Sistema de Información de Tendencias Educativas en América Latina (Siteal) la reducción de las desigualdades entre las zonas urbanas y rurales, y blancos, afrodescendientes o indígenas. Así, puede verse recurrentemente que los sectores históricamente postergados en el acceso a la educación son los grandes beneficiados en momentos de expansión educativa como el que se dio en la región en la última década. ${ }^{1}$

Un aumento generalizado de las tasas de escolarización y una reducción de las brechas de las desigualdades en el acceso a la educación de los adolescentes entre países y dentro de cada país son dos fenómenos que se hacen visibles a partir de una primera lectura de los datos analizados, y que invitan a concluir que el balance de la década en términos de la capacidad de los sistemas educativos de retener en sus aulas a los jóvenes de entre 15 y 17 años de edad es alentador. Sin embargo, hay otros elementos que llevan a relativizar estas buenas noticias. Por un lado, pese a que hubo una clara reducción, aún persisten grandes desigualdades; coexisten en la región países en los que más del $80 \%$ de sus adolescentes están escolarizados con otros en que ese porcentaje no llega al 60\% (tabla 1). Por el otro, en todos los países hay significativas diferencias entre las probabilidades de acceso de estos jóvenes a las escuelas, según su origen social. En casos como en México u 
Honduras, el acceso de los adolescentes de los estratos más altos más que duplica al de los de los estrato bajos.

Es cierto que tras una década que muestra cierto dinamismo en la incorporación de las nuevas generaciones de jóvenes a las escuelas, y en las que el proceso de expansión de las tasas de escolaridad viene acompañado de una reducción de esas disparidades, es posible argumentar que se va avanzando en un camino correcto, ya que la persistencia de estas tendencias en el tiempo va a llevar a la gradual reducción de esas desigualdades que aun hoy persisten en América Latina, y a la universalización del acceso a la educación. Frente a este posible argumento hay advertencias claras respecto a que esta tendencia no necesariamente es posible de ser sostenida en el futuro. En el Informe de Tendencias Sociales y Educativas del Siteal del año 2010 se muestra que el ritmo de expansión de la escolarización en la década que aquí se está analizando fue muy inferior al de la década anterior. Allí puede apreciarse que

(...) durante la década de los años 90 el incremento anual de las tasas específicas de escolarización de la población de entre 5 y 24 años fue de 1.7\%. Esto es, año a año las tasas de escolarización crecieron a ese ritmo, lo cual da para el conjunto de la década un acumulado que se acerca al 20\%. En aquellas edades en que el proceso de expansión estaba menos generalizado, como lo son las correspondientes al nivel inicial o al secundario, el ritmo fue aún mayor; la escolarización de los niños de 5 años creció a un $4.7 \%$ anual, lo cual da para la década un total cercano al 50\%, y entre los adolescentes de 15 a 17 años la expansión se dio a un $2.8 \%$ anual, poco más del 30\% para el decenio. (Siteal, 2010)

El mismo Informe Siteal destaca que, cuando se analiza la primera década de este siglo, lo que se ve es que

(...) el ritmo de expansión decayó sensiblemente. La global se redujo de $1.7 \%$ a $0.6 \%$, sufriendo así una reducción del $65 \%$; a este ritmo, el acumulado para una década no llega al 7\%. El ritmo de expansión de la escolarización entre adolescentes se redujo en un $70 \%$, quedando en un $0.9 \%$; la que menos decreció es la de los niños de 5 años de edad, un $44 \%$. Esto es, en esta última década los avances han sido mucho menos significativos, y esto no responde, como podría esperarse, a que ya se está llegando a la meta de universalización. Por el contrario, ocurre cuando aún queda mucho por avanzar. En el caso de los adolescentes, por ejemplo, aún hay un 25\% desescolarizados. (Ídem, ibíd.)

Aquel documento nos alerta sobre la amenaza del fin de la expansión educativa, al plantear la hipótesis de que los sistemas educativos de la región no están pudiendo superar ciertos techos de escolarización en el camino hacia el acceso universal. Los datos presentados aquí, en la tabla 2, hacen suponer que esa hipótesis no es tan desacertada. Allí puede apreciarse cómo, en ciertos grupos específicos, las tasas mostraron una breve caída. En cuatro países ese fenómeno se ve entre los jóvenes de estratos medios, y en siete países se observa el mismo comportamiento de las tasas 
entre los de los estratos más altos. Esto es, entre aquellos grupos donde las tasas de escolarización ya son relativamente altas, los incrementos son muy leves, nulos e incluso negativos. Y en algunos casos esto ocurre cuando aún esas tasas distan del $100 \%$ de acceso. Se observan en esa tabla ciertas regularidades entre el nivel de las tasas de escolarización al comienzo de la década, y la magnitud del incremento durante esa década. Como ya se destacó, entre los grupos o países que comenzaron el decenio con tasas de escolarización más bajas los incrementos fueron los más significativos, en tanto entre los de las tasas más altas fueron nulos e incluso negativos. Debo aquí reconocer que este hallazgo responde - en parte - a cierta lógica estadística: un país cuya tasa de escolaridad es muy alta tiene poco margen para grandes incrementos, en tanto que aquellos con tasas bajas el rango que los separa del horizonte del acceso universal es mayor, y consecuentemente tiene la probabilidad de mostrar grandes cambios.

Sin embargo, hay otra lógica, que remite a la dinámica de los procesos sociales, que creo relevante destacar aquí. En el año 1990, Rubén Kaztman y Pascual Gerstenfend publicaban en la Revista de la Cepal un artículo titulado "Áreas duras y áreas blandas en el desarrollo social". En aquel trabajo destacaban que las áreas blandas son aquellas cuyos logros no requieren de la movilización de grandes recursos. En las áreas duras, en cambio, quedan comprendidas aquellas que requieren no sólo grandes esfuerzos en términos de movilización de recursos, sino que además, y precisamente por ello, requiere de consensos mucho más difíciles de conseguir, pues apelan a la modificación de aspectos estructurales de la sociedad. Es por ello que el recorrido hacia ciertas metas específicas nunca es lineal, e incluso puede verse interrumpido.

Cuando se analiza la trayectoria de los sistemas educativos hacia la universalización del acceso a la escuela, puede constatarse que en general responde a esta lógica. En el punto inicial de los procesos de expansión, en contextos donde no hay oferta educativa, la tasa de escolarización en establecimientos del sistema formal de instrucción tiende a ser casi nula. Con sólo habilitar un espacio para el dictado de clases y nombrar a docentes las tasas mostrarían un incremento significativo. Ir consolidando una oferta educativa básica es el motor de los primeros pasos de la expansión, y el ritmo de crecimiento de las tasas queda definido por la capacidad que tienen los estados de generar esa oferta.

De todos modos, con ello no alcanza. No todos tendrían acceso a la educación, pues hay otros factores, más allá de la disponibilidad de una oferta básica, que postergan o impiden la escolarización, como por ejemplo aquellas dificultades propias de los hogares en condiciones de pobreza. En estos casos, una política de transferencia de ingresos a los hogares más desventajados tiene, como ya ha quedado demostrado en la región, un impacto significativo. Una vez que están cubierta una oferta 
básica, y resueltos algunos de los factores extraescolares que subyacen a la difícil escolarización, comienzan a aparecer factores que tienen que ver con la calidad de la oferta, sus características, y por último, factores culturales como la valoración que se tiene de la escuela, el lugar que le dan a la educación en sus proyectos de vida las nuevas generaciones, y otros aspectos valorativos comienzan a entrar en juego (Siteal, 2010).

En la medida en que se avanza en este recorrido, las intervenciones necesarias son cada vez más complejas, y la capacidad que se tiene desde el estado en incidir sobre ellas disminuye. Uno de los momentos más críticos de esos procesos se da cuando la oferta educativa pasa a ser mayor que la demanda, esto es, cuando la razón por la que los niños o adolescentes no están escolarizados deja de ser la ausencia de un establecimiento educativo al cual asistir. Quiero entonces enfatizar en este punto que si bien la reducción de los incrementos tiene una lógica puramente estadística, antes señalada, también ese fenómeno responde a dificultades estructurales que terminan consolidando un techo a los procesos de expansión educativa.

Si volvemos a leer la información teniendo presente este proceso, podemos sostener que esa recurrencia encontrada en las tendencias no sólo nos habla de una lógica numérica, sino que además expresan un comportamiento asociado a esa dificultad estructural para lograr la universalización en el acceso al conocimiento. ¿Esa recurrencia expresa una relación lineal? Un análisis de regresiones permite ver que la asociación que hay entre los datos del año 2000 y los del 2010 no es una asociación lineal. El mejor ajuste de esos datos se da con una asociación del tipo cuadrática. Esto es, una curva que expresa precisamente ese proceso de desaceleración de los procesos de expansión. Más aún: dado que el ajuste entre los datos empíricos (aquellos extraídos de las encuestas, y que permitieron hacer este análisis) y el modelo representado por esa curva es muy alto $(\mathrm{r} 2=0.866)$ es posible reconstruir el modelo teórico de las trayectorias que van teniendo los sistemas educativos de la región en su proceso de expansión. Lo que se desprende de este ejercicio de modelización es que el proceso incorporación de adolescentes a las escuelas se encuentra hoy con un techo que está en el $86.7 \%$. El modelo estadístico indica que teniendo como punto de partida una tasa superior a $86.7 \%$ se genera una tendencia negativa, que lleva a que los valores converjan hacia ese punto, comportamiento que no entra en conflicto con lo que puede observarse en las tablas 1 y 2. El gráfico 1 representa la forma que tienen los procesos de expansión en la región, según ese modelo. ${ }^{2}$

¿Qué nos quiere decir esto? El comportamiento de los datos permite predecir que hoy - tal como están las cosas - no es posible llegar al 100\% de escolarización de los adolescentes en la región. Aún en grupos muy privilegiados, como los estratos altos de los países más desarrollados, ese $100 \%$ no se concreta. Cuanto más cerca se está de llegar a esa meta, aparecen variaciones negativas, es decir, hay procesos de 
desescolarización que hablan de ese techo. Si el techo aquí identificado no existiera, los datos analizados se estarían comportando de otro modo.

Pareciera entonces que la universalización del acceso a la escuela por parte de los adolescentes no está siendo posible. Hay aproximadamente un $13 \%$ de adolescentes frente a los cuales actualmente no se cuenta con políticas o prácticas que permitan retenerlos o reinsertarlos a las aulas. Si este techo se consolida, quienes fueron los mayores beneficiados en momentos de expansión educativa, como los jóvenes de las familias de más bajos recursos, los de las zonas rurales, los indígenas, los afroamericanos, y todos aquellos grupos sociales históricamente postergados, pasarán a ser nuevamente los grandes perdedores, quienes quedarán afuera. De todos modos, como se verá más adelante, este techo que hoy se vislumbra en el proceso de expansión educativa va más allá de la dificultad de incorporar plenamente a estos grupos sociales, y expresa además una dificultad estructural de los Estados - y de sus políticas educativas - de interactuar con las nuevas generaciones. Es precisamente esa dificultad la que convierte a la escolarización de los adolescentes en uno de los desafíos más complejos que enfrentan hoy los sistemas educativos de la región.

\section{Identidad y violencia institucional}

¿Quiénes son los adolescentes que quedan en ese núcleo duro que impone un techo al camino hacia el acceso universal? ¿Qué factores intervienen en el "endurecimiento" de la meta de garantizar una educación de calidad para todos? En esta segunda parte del artículo me propongo desarrollar algunas ideas que, lejos de pretender explicar los procesos que subyacen a este estancamiento de la expansión educativa, buscan plantear algunas hipótesis desde las cuales pareciera adecuado abordar su análisis.

Una primera respuesta es, sin duda, las profundas desigualdades económicas vigentes hoy en la región. Ya en la tabla 1 pudo apreciarse las notables diferencias en el acceso de los adolescentes a la escuela según su origen social. Si bien hubo una reducción en la desigualdad entre los diferentes estratos sociales, las brechas oscilan en valores que promedian en un $50 \%$. Esto es, la probabilidad de que un adolescente de estratos sociales altos asista a la escuela es un $50 \%$ más alta que si se trata de uno de estratos bajos. En ciertos países, según se ve en esa misma tabla, puede acercarse y superar el $100 \%$.

Para una familia, lograr que sus niñas y niños puedan permanecer escolarizados desde los primeros años de su vida hasta el final de la adolescencia representa un esfuerzo muy grande, que se traduce regularmente en un costo muy elevado. Más aún, las prácticas educativas presuponen en sí mismas un nivel de bienestar 
básico, cuando se espera que los niños y adolescentes puedan recurrir día a día a las aulas durante más de diez años, y puedan hacer un aprovechamiento activo y enriquecedor de la experiencia escolar. Ellos deben estar sanos, bien alimentados, y deben contar con el tiempo y el espacio necesario para hacer frente al esfuerzo que representa el aprendizaje escolar.

La masificación de la escuela media, y los avances hacia el horizonte de universalización de ese nivel hizo que cada vez más ingresen a las aulas adolescentes provenientes de los estratos sociales más bajos, de familias que no cuentan con las condiciones de bienestar necesarias, y donde ellos mismos se ven involucrados en la producción de ingresos para el hogar. Expresión de ello es la alta presencia de adolescente que trabajan en el universo de los escolarizados. La tabla 3 muestra que entre los adolescentes escolarizados de entre 15 y 17 años provenientes de estratos sociales más bajo valores que promedian el $24 \%$ son además económicamente activos, llegando en algunos países como Bolivia o Perú a representar una gran mayoría. Estos valores superan significativamente a los de los provenientes de los sectores sociales más altos. Pero en situaciones más extremas, la escolarización se les hace imposible. O porque las familias no pueden renunciar a los ingresos que representa la presencia de estos adolescentes insertos plenamente en el mundo del trabajo (habitualmente los varones) o porque no pueden renunciar a las funciones de cuidado de los niños menores, o de ancianos en el hogar (responsabilidad que cae habitualmente sobre las adolescentes mujeres). ${ }^{3}$ No hay dudas de que los efectos de la desigualdad ingresaron a las aulas.

De todos modos, si bien es clara la relación entre el nivel socioeconómico y la escolarización, en la tabla 1 puede apreciarse también que entre los adolescentes de los sectores medios y altos hay niveles significativos de desescolarización. En el Informe de Tendencias Sociales y Educativas que el Siteal publicó en el año 2008, dedicado a analizar la relación de la escuela con los adolescentes, se destaca que, para el grupo específico de 17 años de edad, aproximadamente la mitad de los desescolarizados no son pobres. Esto es, se sugiere allí que la dimensión económica permite comprender la mitad del problema de la desescolarización de los adolescentes. ¿Qué pasa con la otra mitad? (Siteal, 2008).

Es que no sólo las desigualdades sociales entraron a las aulas de la mano del proceso de masificación de la escuela media que se está viviendo en la región. También se hace visible hoy como nunca al interior de las escuelas medias la compleja diversidad que configura el escenario social actual. Esa diversidad se expresa de muchos modos: adolescentes y jóvenes provenientes de los pueblos indígenas o afrodescendientes que antes no ingresaban a las escuelas, y ahora están en ellas, o alumnos que provienen de otros países, con otras culturas y lenguas. También es expresión de la diversidad que irrumpe en las aulas la presencia de adolescentes que 
construyen su identidad desde representaciones y referentes muy diversos, visibles en sus modos de vestir, en sus consumos culturales, en sus preferencias sexuales, o en su modo de leer e interpretar el mundo.

En el IIPE-Unesco en Buenos Aires venimos realizando desde el año 2001 un conjunto de investigaciones orientadas a desentrañar los mecanismos a través de los cuales se van construyendo estas desigualdades educativas. Allí se ve claramente que desigualdad y diversidad se articulan, y como resultado de ello, los alumnos que hoy participan de las prácticas educativas en las escuelas medias de la región poco se parecen a aquel alumno para el cual esas escuelas fueron concebidas, o para quienes fueron formados sus docentes. Por un lado, son diferentes por ser muchos de ellos más pobres que aquel alumno esperado. En otro caso, por ser indígenas, afroamericanos, migrantes, o por presentarse como emos, punks o góticos. En entrevistas realizadas en esos estudios pudo constatarse que muchos docentes, a la hora de explicar el fracaso escolar de sus alumnos, suelen poner de manifiesto otros rasgos - además de aquellos que nos hablan de la desigualdad o la diversidad - de aquel alumno para el cual están trabajando. Lo hacen al dar lugar a expresiones del tipo "...y cómo no le va a ir mal si su familia no lo apoya en nada...", o "...cómo va a aprender si su padre está preso...". ${ }^{4}$

De este modo es posible sostener, a partir de un análisis cuidadoso de esos discursos tan vigentes, que el alumno que está en la representación de muchos de los docentes y directivos en el momento de planificar y concebir sus dinámicas institucionales y sus prácticas es un alumno urbano, blanco, de clase media o media alta, de familia "bien constituida" y de "moral intachable". ${ }^{5}$ Marisa Stigaard, una de las investigadoras del equipo que realizó estos estudios, al analizar en detalle entrevistas a docentes de escuelas del nivel medio realizadas en cuatro países de América Latina, concluye que el alumno deseado por los docentes es aquel que

(...) sabe hacer su trabajo como estudiante, es autónomo en responder a sus aprendizajes, realiza sus trabajos, lee bastante, sabe proyectarse hacia el futuro, no necesita que nadie le diga que debe hacer, es consciente del papel que tiene como estudiante y como ser social, tiene interés en mejorar, en superarse, es solidario, democrático, resolutivo, emprendedor, puntual, cuenta con el apoyo de su familia, es comprometido, activo, partícipe, disciplinado, aplicado y respetuoso. (Stigaard, 2012, p. 255)

Esa diversidad de adolescentes y jóvenes que hoy ingresan a las aulas de las escuelas medias va generando un creciente desajuste en términos valorativos entre el alumno que se quisiera tener, y aquel que efectivamente está en el aula día a día. No sólo se constata que los nuevos alumnos son diferentes, sino además se hace visible que esa diferencia genera malestar, y más aún, es connotada y valorada negativamente. 
Muchas veces el alumno real, aquel que ingresa diariamente a las aulas, es despreciado, su identidad no interesa, es escasa la voluntad de establecer con él un diálogo basado en su reconocimiento. Por el contrario, frente a ese nuevo alumno real - ese alumno "atípico" - persiste la esperanza de convertirlo en el alumno deseado, y desde esa lógica es visible cómo se premian los esfuerzos que cada niño o adolescente hace por aceptar esa transformación. Para un adolescente, permanecer en la escuela significa aceptar el juego de actuar un personaje que no es, ocultar su compleja identidad, reprimir la posibilidad de experimentar o desplegar nuevas narrativas sobre sí mismo. La irrupción de rasgos que no son compatibles con la imagen idealizada por sus docentes puede significarle la aparición de un sinnúmero de estrategias institucionales de sanción y exclusión (López, 2012a).

Esta tensión ya no es expresión de la desigualdad, ni es expresión de la diversidad, sino que da cuenta de cierto desprecio por aquellos cuyo origen social o cuya identidad difieren de aquel alumno añorado. Los ejemplos son múltiples: la docente que considera que algunos de sus alumnos son como "la manzana podrida", que si no se la saca a tiempo contagia a las demás, aquel maestro que en una entrevista manifestaba que le molestaba el olor de sus alumnos, el directivo que manifiesta que "con lo que me costó expulsar a ese joven, ahora me obligan a recibirlo nuevamente, y encima con beca...", la docente que considera que la escuela no es para "esta clase de chicos". Quienes no responden a la imagen de aquel alumno ideal se ven diariamente violentados por las instituciones educativas, las cuales se constituyen en instituciones que, operando bajo el mandato de la inclusión educativa, terminan asumiendo prácticas claramente discriminatorias. Adquiere aquí sentido la hipótesis de que una de las principales causas de desescolarización de los adolescentes, o de la dificultad de seguir avanzando en la expansión de la educación media, está en la violencia que muchas instituciones tienen hacia ellos al no estar dispuestas a establecer un diálogo que parta de reconocer quiénes son, de asumir su verdadera identidad.

Es posible en este punto proponer hipótesis que van más allá del ámbito escolar, y reconocer que esta tendencia a negar la identidad de los alumnos, y en especial la de los adolescentes, refleja un proceso que va excede al sistema educativo mismo, y que puede ser analizada como expresión de la dificultad del propio Estado de generar una relación con adolescentes y jóvenes basada en su reconocimiento. Lejos de promover para ellos un espacio de crecimiento, experimentación y despliegue de las identidades, el Estado suele establecer una relación con los adolescentes y jóvenes que parte del supuesto de que ellos deben ser encauzados. Son vistos como sujetos carentes, en transición, preparándose para ser adultos, que deben ser recuperados, orientados, que no se puede confiar en ellos (López, 2012b; Stigaard 2012). 
Subyace a esta lógica un conjunto de representaciones instaladas en la sociedad, sumamente naturalizadas, que los Estados reproducen en sus prácticas. Durante el trabajo de campo que realizó para su tesis doctoral, Mariana Chaves identificó un conjunto de representaciones en torno al ser jóvenes, entre las que se destacan las siguientes: el joven como ser inseguro de sí mismo, como ser en transición, como ser no productivo, como ser incompleto, como ser desinteresado y/o sin deseo, como ser desviado, como ser peligroso, como ser rebelde o peligroso, o como ser del futuro (Chaves, 2006).

Como efecto de ello, la relación que se establece con ellos debe ser una relación basada en el principio de orientación, represión, y - tal como se propuso aquí - negación. No importa cómo son hoy, pues hoy son carentes, peligrosos, y están desorientados. Lo que importa es cómo deben ser. Y frente a este razonamiento, las instituciones estatales se muestran conocedoras de ese "cómo deben ser". ¿Cómo deben ser? Desde la escuela se dan señales claras al respecto. Deben ser como ese alumno idealizado, aquel responsable, comprometido, solidario o resolutivo. Deben ser de moral intachable, y - en lo posible - urbanos, blancos y de clase media. Estas señales, tal como se pudo ver, las instituciones educativas las dan a través de sus prácticas diarias, de la valoración que hacen de sus alumnos, de lo que premian y castigan, o través de su currículum (López, 2012a).

\section{Comentario final}

El análisis estadístico realizado en la primera parte del artículo instala fuertemente la posibilidad de pensar en que, tal como están funcionando hoy los sistemas educativos y como se articulan con las demandas y características de la sociedad latinoamericana, la meta de universalización de la escolarización para los adolescentes es aún inviable. Llegando al $90 \%$ de escolarización, los avances registrados son ínfimos, e incluso negativos.

Tal como se mencionó más adelante, este estancamiento de los procesos de expansión educativa se da en contextos donde cabe suponer que la oferta educativa es amplia, donde se puede sostener que no es la falta de escuelas lo que impone ese límite. Es por ello que aquí se avanza en proponer otras hipótesis que invitan a poner la mirada en la dinámica de esos establecimientos educativos, en el modo en que proponen relacionarse con la sociedad en su conjunto, y con los adolescentes en particular.

La persistencia de profundas desigualdades en la región, las crecientes expresiones de su diversidad cultural e identitaria, y las nuevas formas de posicionamiento de los ciudadanos ante las acciones de los Estados - exigiendo, entre otras 
cosas, un trato basado en el reconocimiento - reconfiguran de modo estructural el contexto y las condiciones en que tienen lugar las prácticas educativas, y llevan a la necesidad de rediseñar a fondo, en especial desde las instituciones de la escuela media, el modo en que se aborda el compromiso público de garantizar a las nuevas generaciones una educación de calidad para todos.

\section{Notas}

1. Se invita aquí a ver los diferentes Informes de Siteal (www.siteal.iipe-oei.org), donde se analiza en profundidad esta reducción de las desigualdades.

2. Una descripción detallada del ejercicio estadístico desde el cual se llega a esta conclusión está desarrollada en el anexo 2, al final del artículo.

3. Este punto está especialmente desarrollado en el texto "Adolescentes que no estudian ni trabajan", de Vanesa D'Alessandre, publicado en la serie Cuadernos del Siteal (http://www.siteal.org/cuadernos/10/adolescentes-que-no-estudian-ni-trabajan).

4. Se hace referencia al estudio "Educación, reformas y equidad en los países del Cono Sur", que IIPE-Unesco (Buenos Aires) realiza desde el año 2001 con el apoyo de la Fundación Ford.

5. Esta relación entre alumno ideal y alumno real fue desarrollada más en profundidad en el libro Equidad educativa y desigualdad social (López, 2005).

\section{Referencias}

CHAVES, M. Informe Investigaciones sobre Juventud en la Argentina, estado del arte en ciencias sociales 1983-2006. Revista Electrónica del Instituto de Altos Estudios Sociales de la Universidad Nacional de General San Martín, Buenos Aires, v. 2, n. 5, jun. 2006.

KAZTMAN, R.; GERSTENFELD, P. Áreas duras y áreas blandas en el desarrollo social. Revista de la Cepal, Santiago de Chile, n. 41, LC/G.1631-P, ago. 1990.

LÓPEZ, N. Equidad educativa y desigualdad social: desafíos a la educación en el nuevo escenario latinoamericano. Buenos Aires: IIPE-Unesco, 2005.

LÓPEZ, N. El desprecio por ese alumno. In: LÓPEZ, N. (Coord.). Escuela, identidad y discriminación. Buenos Aires: IIPE-Unesco, 2012a.

LÓPEZ, N. Equidad educativa y diversidad social. Buenos Aires: IIPE-Unesco, 2012b.

SITEAL. Informe sobre Tendencias Sociales y Educativas en América Latina. Buenos Aires: IIPE-Unesco; OEI, 2008.

SITEAL. Informe sobre Tendencias Sociales y Educativas en América Latina. Buenos Aires: IIPE-Unesco; OEI, 2010. 
Adolescentes en las aulas: la irrupción de la diferencia y el fin de la expansión educativa

STIGAARD, M. El Estado y la inclusión educativa de los jóvenes frente a la injusticia distributiva y cultural. In: LÓPEZ, N. Equidad educativa y diversidad social. Buenos Aires: IIPE-Unesco, 2012.

Recebido em 3 de junho de 2012.

Aprovado em 25 de julho de 2012. 


\section{ANEXOS}

\section{I - Tablas y gráficos}

\section{Tabla 1}

Tasas específicas de escolarización de adolescentes de 15 a 17 años de edad, según nivel socioeconómico. 18 países de América Latina (2000-2010).

\begin{tabular}{|c|c|c|c|c|c|c|c|c|}
\hline & \multicolumn{4}{|c|}{ Cerca de 2000} & \multicolumn{4}{|c|}{ Cerca de 2010} \\
\hline & \multirow[b]{2}{*}{ Total } & \multicolumn{3}{|c|}{ Nivel socioeconómico } & \multirow[b]{2}{*}{ Total } & \multicolumn{3}{|c|}{ Nivel socioeconómico } \\
\hline & & Bajo & Medio & Alto & & Bajo & Medio & Alto \\
\hline Argentina Urbano & 85.2 & 66.9 & 84.4 & 97.3 & 87.8 & 75.6 & 84.8 & 95.0 \\
\hline Bolívia & 76.4 & 55.4 & 88.8 & 88.5 & 86.8 & 79.0 & 90.4 & 96.4 \\
\hline Brasil & 81.1 & 74.5 & 85.5 & 90.8 & 85.2 & 80.1 & 87.5 & 91.9 \\
\hline Colombia & 67.8 & 53.7 & 76.0 & 87.5 & 75.5 & 67.3 & 79.0 & 86.4 \\
\hline Costa Rica & 58.2 & 35.1 & 62.4 & 92.7 & 79.5 & 58.4 & 82.4 & 96.7 \\
\hline Chile & 87.8 & 71.1 & 86.6 & 98.1 & 91.4 & 82.2 & 90.0 & 95.3 \\
\hline República Dominicana & 83.8 & 74.1 & 89.6 & 96.6 & 84.5 & 76.2 & 85.9 & 94.9 \\
\hline Ecuador & 63.8 & 35.8 & 73.6 & 89.2 & 76.5 & 56.8 & 78.3 & 93.7 \\
\hline El Salvador & 62.8 & 48.5 & 80.0 & 92.7 & 70.8 & 57.3 & 81.2 & 96.7 \\
\hline Guatemala & 40.7 & 31.9 & 71.4 & 86.6 & 56.9 & 46.8 & 84.8 & 92.4 \\
\hline Honduras & 44.1 & 28.5 & 68.4 & 85.1 & 53.5 & 36.8 & 71.5 & 88.4 \\
\hline México & 57.9 & 38.8 & 66.0 & 90.6 & 66.2 & 43.8 & 67.0 & 91.5 \\
\hline Nicaragua & 58.9 & 46.3 & 77.7 & 81.7 & 57.7 & 44.7 & 73.2 & 75.1 \\
\hline Panama & 75.1 & 46.0 & 78.0 & 90.7 & 79.9 & 54.7 & 81.8 & 94.7 \\
\hline Paraguay & 63.8 & 43.3 & 77.5 & 92.0 & 71.1 & 56.2 & 75.8 & 85.3 \\
\hline Perú & 69.9 & 57.2 & 76.7 & 73.3 & 84.0 & 71.7 & 86.8 & 93.4 \\
\hline Uruguay & 77.2 & 50.2 & 76.6 & 97.0 & 77.2 & 57.7 & 76.2 & 95.5 \\
\hline Venezuela & 68.6 & 45.7 & 75.6 & 90.8 & 80.3 & 62.2 & 81.5 & 92.2 \\
\hline Total(*) & 68.0 & 50.2 & 77.5 & 90.1 & 75.8 & 61.5 & 81.0 & 92.0 \\
\hline
\end{tabular}

Fuente: Elaboración propia, en base datos de Siteal.

(*) El total está calculado a partir de un promedio no ponderado de los valores que la variable toma en cada uno de los países. Al no estar ponderado por el peso poblacional de cada país, el valor que toma este total no expresa una tasa de escolarización de la región, sino un valor hacia el cual tienden el conjunto de las tasas de cada uno de los países. 
Tabla 2

Variación de las tasas específicas de escolarización de adolescentes de 15 a 17 años de edad entre 2000 y 2010, según nivel socioeconómico. 18 países de América Latina.

\begin{tabular}{|l|c|c|c|c|}
\hline \multirow{2}{*}{} & \multicolumn{4}{c|}{ Nivel socioeconómico } \\
\cline { 2 - 5 } & Total & Bajo & Medio & Alto \\
\hline Argentina Urbano & $3.1 \%$ & $13.0 \%$ & $0.5 \%$ & $-2.4 \%$ \\
\hline Bolívia & $13.6 \%$ & $42.6 \%$ & $1.8 \%$ & $8.9 \%$ \\
\hline Brasil & $5.1 \%$ & $7.5 \%$ & $2.3 \%$ & $1.2 \%$ \\
\hline Colombia & $11.4 \%$ & $25.3 \%$ & $3.9 \%$ & $-1.3 \%$ \\
\hline Costa Rica & $36.6 \%$ & $66.4 \%$ & $32.1 \%$ & $4.3 \%$ \\
\hline Chile & $4.1 \%$ & $15.6 \%$ & $3.9 \%$ & $-2.9 \%$ \\
\hline República Dominicana & $0.8 \%$ & $2.8 \%$ & $-4.1 \%$ & $-1.8 \%$ \\
\hline Ecuador & $19.9 \%$ & $58.7 \%$ & $6.4 \%$ & $5.0 \%$ \\
\hline El Salvador & $12.7 \%$ & $18.1 \%$ & $1.5 \%$ & $4.3 \%$ \\
\hline Guatemala & $39.8 \%$ & $46.7 \%$ & $18.8 \%$ & $6.7 \%$ \\
\hline Honduras & $21.3 \%$ & $29.1 \%$ & $4.5 \%$ & $3.9 \%$ \\
\hline México & $14.3 \%$ & $12.9 \%$ & $1.5 \%$ & $1.0 \%$ \\
\hline Nicaragua & $11.4 \%$ & $29.8 \%$ & $-2.2 \%$ & $-7.3 \%$ \\
\hline Panama & $-2.0 \%$ & $-3.5 \%$ & $-5.8 \%$ & $-8.1 \%$ \\
\hline Paraguay & $11.6 \%$ & $25.3 \%$ & $13.2 \%$ & $27.4 \%$ \\
\hline Perú & $0.0 \%$ & $14.9 \%$ & $-0.5 \%$ & $-1.5 \%$ \\
\hline Uruguay & $22.6 \%$ & $4.5 \%$ & $2.1 \%$ \\
\hline Venezuela & & $18.9 \%$ & $4.9 \%$ & $4.4 \%$ \\
\hline Total (No ponderado) & $56.1 \%$ & $7.8 \%$ & \\
\hline & $17 \%$ & & \\
\hline
\end{tabular}

Fuente: Elaboración propia, en base datos de Siteal. 
Tabla 3

Brechas de escolarización entre adolescentes de 15 a 17 años de edad de nivel socioeconómico alto y bajo, y variación de esas brechas entre 2000 y 2010. 18 países de América Latina (2000-2010).

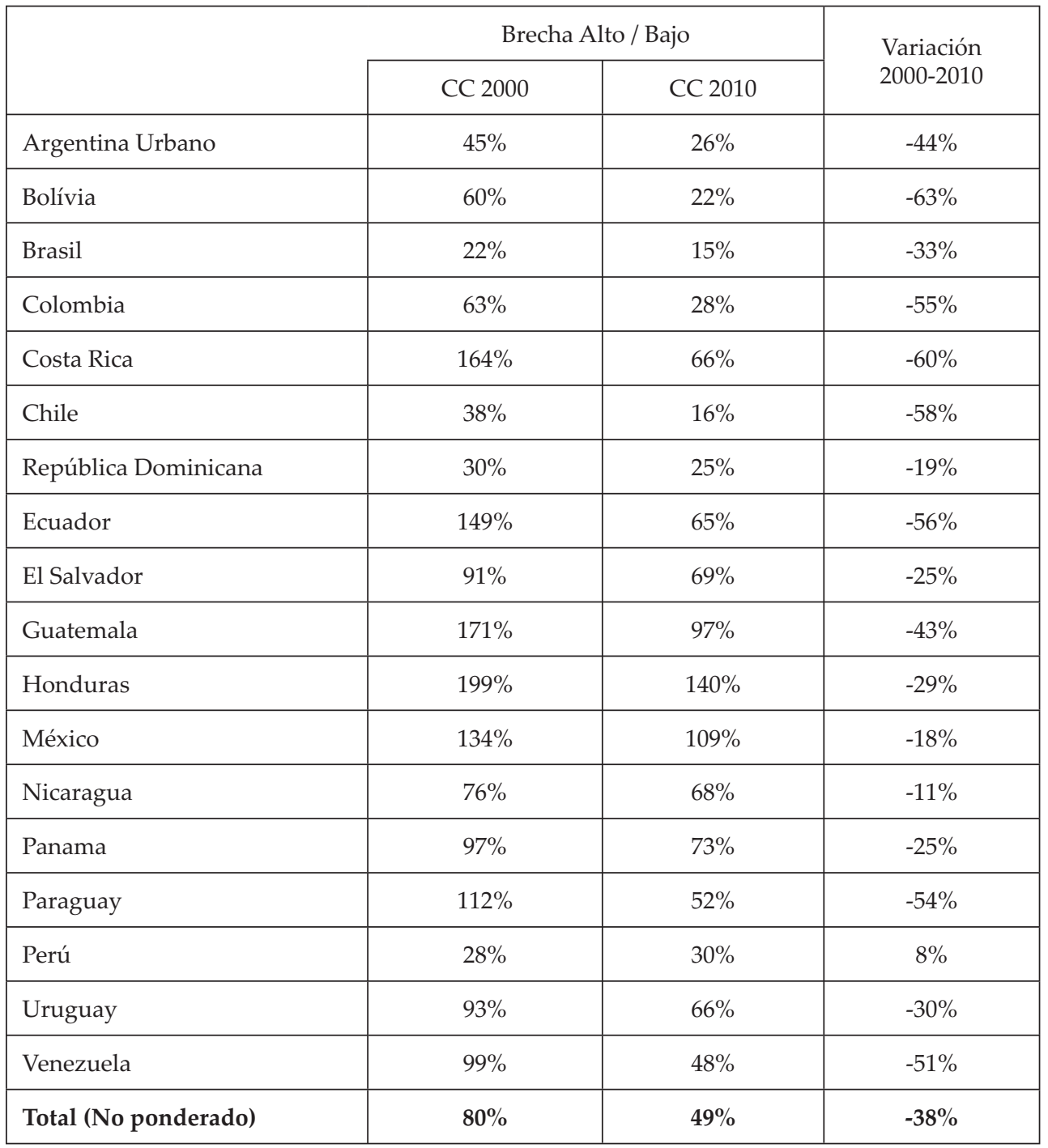

Fuente: Elaboración propia, en base datos de Siteal. 


\section{Tabla 4}

Tasa de actividad económica entre adolescentes escolarizados de 17 a 17 años de edad, según nivel socioeconómico, y brecha entre las tasas de de los estratos sociales altos y bajos.

18 países de América Latina (2010).

\begin{tabular}{|c|c|c|c|c|}
\hline & & cioeco & & Brecha \\
\hline & B & $\mathrm{M}$ & A & NSE Alto / NSE Bajo \\
\hline Argentina Urbano & $3 \%$ & $6 \%$ & $3 \%$ & 1.0 \\
\hline Bolívia & $43 \%$ & $28 \%$ & $18 \%$ & 2.4 \\
\hline Brasil & $37 \%$ & $32 \%$ & $24 \%$ & 1.5 \\
\hline Colombia & $14 \%$ & $10 \%$ & $7 \%$ & 2.1 \\
\hline Costa Rica & $9 \%$ & $7 \%$ & $2 \%$ & 3.9 \\
\hline Chile & $6 \%$ & $5 \%$ & $5 \%$ & 1.3 \\
\hline República Dominicana & $16 \%$ & $10 \%$ & $9 \%$ & 1.8 \\
\hline Ecuador & $24 \%$ & $14 \%$ & $7 \%$ & 3.6 \\
\hline El Salvador & $21 \%$ & $14 \%$ & $4 \%$ & 5.2 \\
\hline Guatemala & $28 \%$ & $17 \%$ & $10 \%$ & 2.9 \\
\hline Honduras & $24 \%$ & $16 \%$ & $10 \%$ & 2.5 \\
\hline México & $16 \%$ & $14 \%$ & $9 \%$ & 1.9 \\
\hline Nicaragua & $30 \%$ & $13 \%$ & $15 \%$ & 2.0 \\
\hline Panama & $27 \%$ & $9 \%$ & $3 \%$ & 8.4 \\
\hline Paraguay & $41 \%$ & $33 \%$ & $17 \%$ & 2.4 \\
\hline Perú & $63 \%$ & $45 \%$ & $23 \%$ & 2.7 \\
\hline Uruguay & $18 \%$ & $15 \%$ & $8 \%$ & 2.3 \\
\hline Venezuela & $11 \%$ & $7 \%$ & $4 \%$ & 2.6 \\
\hline Total (No ponderado) & $24 \%$ & $16 \%$ & $10 \%$ & 2.4 \\
\hline
\end{tabular}

Fuente: Elaboración propia, en base datos de Siteal. 


\section{Gráfico 1}

Modelo teórico de expansión de la tasa de escolarización de los adolescentes de 15 a 17 años de escolaridad en América Latina, a partir de datos de 18 países de la región (2000-2010).

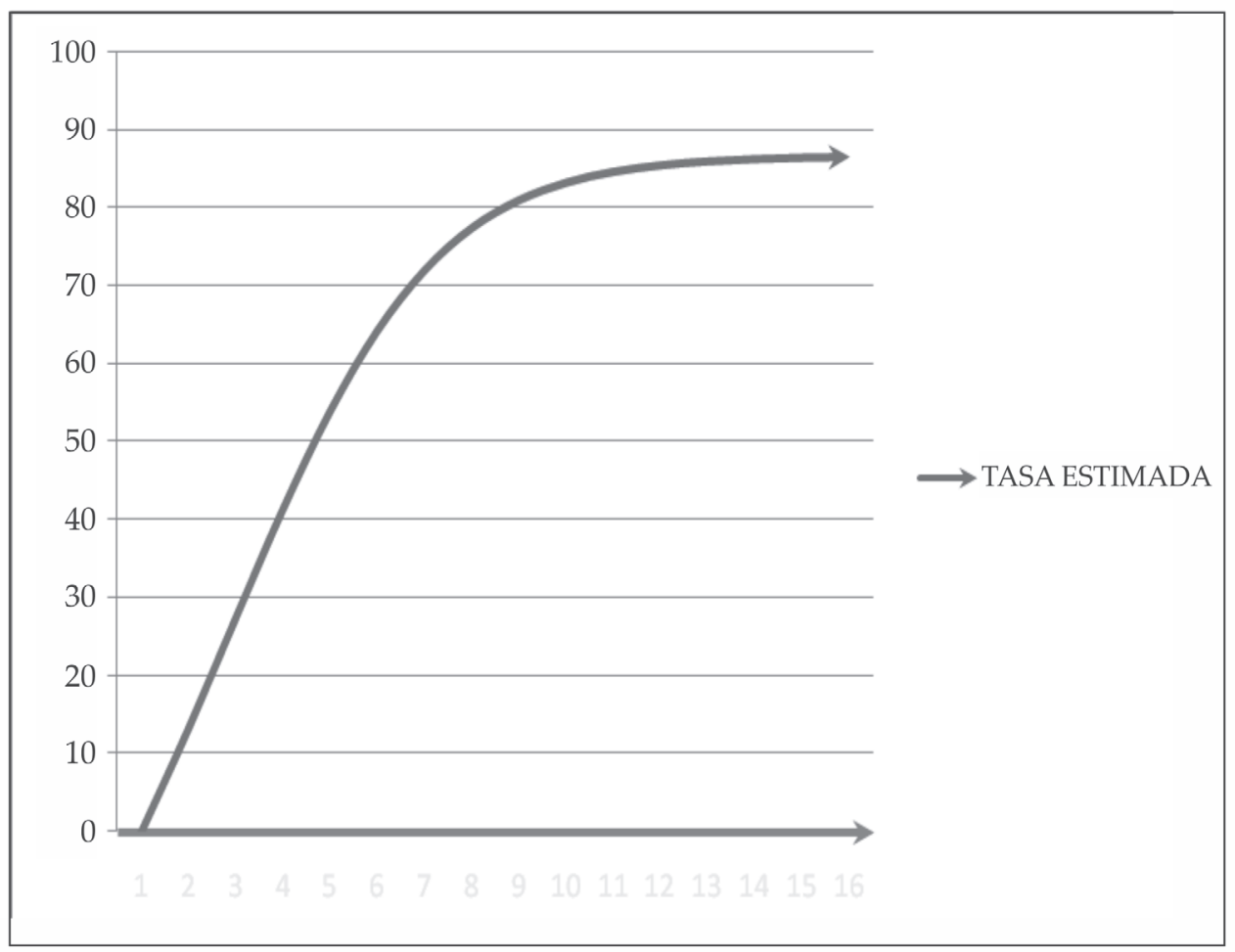


Adolescentes en las aulas: la irrupción de la diferencia y el fin de la expansión educativa

Bases de datos utilizadas por el Proyecto Siteal para la producción de los datos aquí analizados.

\begin{tabular}{|c|c|c|c|}
\hline & CC 2000 & CC 2010 & Fuente \\
\hline Argentina Urbano & 2000 & 2010 & EPH del Indec \\
\hline Bolivia & 2000 & 2009 & ECH del INE \\
\hline Brasil & 2001 & 2009 & PNAD del IBGE \\
\hline Colombia & 2003 & 2010 & ECH del Dane \\
\hline Costa Rica & 2000 & 2010 & EHPM del Inec \\
\hline Chile & 2000 & 2009 & Casen de Mideplan \\
\hline República Dominicana & 2000 & 2010 & $\begin{array}{l}\text { ENFT del Banco Central de la República } \\
\text { Dominicana }\end{array}$ \\
\hline Ecuador & 2001 & 2009 & EESD del Inec \\
\hline El Salvador & 2000 & 2009 & EHPM de la Digestyc \\
\hline Guatemala & 2001 & 2010 & 2001 ECV del INE, 2010 Enei del INE \\
\hline Honduras & 2001 & 2009 & EPHPM del INE \\
\hline México & 2000 & 2010 & Enigh del Inegi \\
\hline Nicaragua & 2001 & 2009 & EMNV del Inec \\
\hline Panama & 2000 & 2010 & ECH del INE \\
\hline Paraguay & 2000 & 2010 & EIDH de la DGEEC \\
\hline Perú & 2000 & 2009 & ENH del Inei \\
\hline Uruguay & 2001 & 2009 & ECH del DEC \\
\hline Venezuela & 2000 & 2009 & EH del INE \\
\hline
\end{tabular}

Fuente: Siteal (www.siteal.org). 


\section{II - Cálculo de la tasa estimada de escolarización de los adolescen- tes de 15 a 17 años en el tiempo, y del valor del techo de expansión.}

El ejercicio de estimación de valores futuros de las tasas de escolarización de los adolescentes de 13 a 17 años de edad se basa en un cálculo de regresión. El insumo de ese ejercicio fueron los 72 pares ordenados de valores que resultan de tomar, en la tabla 1, una tasa de escolarización para el año 2000 y su equivalente para el 2010.

El supuesto que hay detrás de esta elección es que hay una lógica en el valor que toma la tasa de escolarización en el 2010, dependiente del valor que el mismo grupo tenía en el 2000. Esto es, la magnitud de los incrementos durante el período queda predefinida por el valor inicial.

Un análisis de regresiones de esos valores nos ofrece el siguiente resultado:

\section{Resumen del modelo y estimaciones de los parámetros}

Variable dependiente: Valor Final.

\begin{tabular}{|l|c|c|c|c|c|c|c|c|}
\hline \multirow{2}{*}{ Ecuación } & \multicolumn{4}{|c|}{ Resumen del modelo } & \multicolumn{3}{c|}{ Estimaciones de los parámetros } \\
\cline { 2 - 9 } & R cuadrado & $\mathrm{F}$ & $\mathrm{gl1}$ & $\mathrm{gl}$ & Sig. & Constante & $\mathrm{b} 1$ & $\mathrm{~b} 2$ \\
\hline Lineal & .862 & 463.783 & 1 & 74 & .000 & 24.227 & .747 & \\
Cuadrático & .866 & 236.398 & 2 & 73 & .000 & 13.276 & 1.107 & -.003 \\
Compuesto & .835 & 374.355 & 1 & 74 & .000 & 35.357 & 1.011 & \\
Crecimiento & .835 & 374.355 & 1 & 74 & .000 & 3.566 & .011 & \\
\hline
\end{tabular}

La variable independiente es Valor Inicial.

Allí se aprecia que el mayor ajuste se da con una ecuación cuadrática, la cual toma la siguiente forma, si se consideran los parámetros estimados

$$
\text { Valor Final }=1.107 * \text { Valor Inicial }-.003 \text { Valor Inicial } 2+13.276
$$

Para reproducir la curva de tendencia presentada en el gráfico 1, se utilizó el siguiente procedimiento: en un momento T0 se supuso una tasa de escolarización inicial 0. Pasado un período de tiempo se llega a T1, y según la ecuación anterior en ese momento el valor que resulta es 13.28. Tomando ahora como valor inicial 13.28, se aprecia que en un tiempo T2 la tasa de escolarización será de 27.44. Repitiendo esta lógica recurrentemente se va reconstruyendo la trayectoria de la tasa de escolarización modelizada en el tiempo, y se constata que a partir de 86.70 no se aprecian más incrementos. 